\title{
6 Die Frage nach dem Ursprung der ludi saeculares und ihr Mythos
}

Die Erforschung des Urspungs der Säkularfeier in Rom stößt trotz der sehr guten Quellenlage zur augusteischen Säkularfeier auf erhebliche Schwierigkeiten. Während man die Gestaltung und Aussage der augusteischen Feier aufgrund des commentarium sehr genau rekonstruieren kann, ist es nicht möglich, ähnlich präzise Aussagen über Gestaltung und Aussage früherer Säkularfeiern zu machen und auf diese Weise eine Entwicklung nachzuzeichnen. Es liegen zwar Quellen über frühere Feiern vor, doch stammen sie ausnahmslos von späteren Autoren, die alle erst nach der augusteischen Säkularfeier geschrieben haben. ${ }^{1}$ Diese Quellen überliefern möglicherweise gewisse Informationen über irgendwelche Feiern nur deshalb, weil sie darin Ähnlichkeiten mit der augusteischen Feier sahen.

Dennoch ist in der Geschichte der Erforschung der ludi saeculares bis in die jüngste Zeit immer wieder versucht worden, eine Entwicklungslinie der Säkularspiele zu konstruieren. Die wichtigsten dieser Versuche seien hier kurz skizziert:

Ausgehend von der Darstellung der Spiele des Valerius Poplicola bei Plutarch (Poplic. 2I) stellt Coarelli, dessen Aussagen auf Wagenvoort beruhen, einen $\mathrm{Zu}$ sammenhang zwischen dem Austragungsort dieser Spiele und den ludi saeculares her, weil beide im Marsfeld stattfanden. ${ }^{2}$ Außerdem schreibt die Überlieferung der gens Valeria eine besondere Beziehung zum Marsfeld zu, die angeblich durch den Fund des lapis Satricanus eine Bestätigung fand, weil dieser Stein eine Weihinschrift eines Valeriers an den Gott Mars enthält. ${ }^{3}$ Nach diesen Annahmen liegt es unter Berücksichtigung des überlieferten Ursprungsmythos der ludi saeculares nicht fern, anzunehmen, dass ein ursprünglich dem Mars geltender Gentilkult der

I Als Hauptquellen sind zu werten: Zos. 2, I-6; Val. Max. 2, 4; Cens. I7; Plut. Poplic. 2 I. Vgl. Tabelle S. I 70 f.

2 Wagenvoort (1956); Coarelli (i997) i I $2 \mathrm{ff}$.

3 Nachdem Tarquinius sich das Marsfeld als Privatbesitz angeeignet hatte, obwohl es dem Gott Mars geweiht war, wurde unter dem Konsulat des Valerius Poplicola nach der Beseitigung der Königsherrschaft diese als frevelhaft empfundene Aneignung wieder rückgängig gemacht (Dion. Hal. ant. 5, I 3, 2 f.; Liv. 2, 5, I ff.). Die erneute Weihung des Gebietes an Mars gab dem Ort seine Bedeutung als sakralem, öffentlichem Raum zurück, die der Ort offenbar schon in der Königszeit als Versammlungsplatz des Volkes zur Wahl des Königs und der Magistrate hatte. Dagegen gibt Plut. Poplic. 8, I an, dass das Gebiet erst unter Poplicola dem Mars geweiht worden sei. Zum lapis Satricanus liegen zwei Interpretationen vor: Versnel (1982) 193-235; Coarelli (1997) I I ff. Während Versnel eine Identifikation des Valeriers der Inschrift mit Valerius Poplicola für nicht gesichert hält, versucht Coarelli seine These zu stärken, dass ein Gentilkult der Valerier auf dem Marsfeld dem Gott Mars gegolten habe. Somit hätte ein Gentilkult der Valerier dem Ort den Namen als campus Martius gegeben. Damit folgt er Wagenvoort, der schon vor dem Fund des lapis Satricanus überzeugt war, dass die ludi saeculares 
Valerier, der im Marsfeld angesiedelt war, der Ausgangspunkt für die späteren ludi saeculares gewesen sein könnte. Im Wesentlichen stützt sich diese These auf die Angaben Plutarchs über die Spiele des Poplicola. ${ }^{4}$ Eine Verbindung der gens Valeria mit dem Marskult mag bestanden haben, aber der lapis Satricanus beweist eine solche nur für die Stadt Satricum, nicht für Rom. Der Gott Mars ist außerdem an den Säkularspielen in keiner Weise erwähnt. Ebenso unbeweisbar ist, dass die Spiele des Poplicola irgendetwas mit Säkularspielen zu tun hatten. Gewisse Riten dieser Spiele weisen unzweifelhaft auf diejenigen der Säkularspiele hin, es ist aber nicht möglich, herauszufinden, ob diese Spiele deswegen nachträglich in die Tradition der Säkularspiele gestellt wurden, geschweige denn, ob es sich um eine Ursprungsform von Säkularspielen handelt. Bei all diesen Untersuchungen handelt sich um Spekulationen, die durch Verknüpfungen mit zum Teil fernliegenden Informationen eine scheinbare Plausibilität erhalten.' Dabei wächst der Erkenntnisgewinn für die ludi saeculares nicht, denn es bleibt nach wie vor ungewiss, wie und unter welchen Voraussetzungen solche frühen Feiern abgehalten wurden. Im Gegenteil erfordern solche Spekulationen immer wieder neue Annahmen, die zum Teil hinter den einmal erreichten Wissensstand zurückgehen.

Dies ist der Fall für das Verständnis des Gottes Mars. Um eine Linie zwischen den augusteischen Säkularspielen, die das Hauptgewicht auf Fruchtbarkeit legen, und der als erster Säkularfeier geltenden Feier des Poplicola herstellen zu können, ist es nötig, auch den Marskult in irgendeiner Weise als Fruchtbarkeitskult zu

sehr viel älter seien als die ludi Terentini von 249 v. Chr. und auf Zusammenhänge zwischen dem Ursprungsmythos der ludi saeculares und gewisse Elemente des Marskultes hinwies. Als Stütze für seine Vermutungen führt Wagenvoort Abbildungen auf etruskischen Spiegeln an, die er als Wiedergeburtsmythos des Mars interpretiert. Weitere Argumente Wagenvoorts, um eine Verbindung des Mars mit dem Marsfeld zu stützen, sind: der unterirdische Altar auf dem Terentum, die dort abgehaltenen Pferderennen und die im Mythos der ludi saeculares hervorgehobene Bedeutung der Heilung durch das erwärmte Tiberwasser, was wiederum den Bezug zu den etruskischen Spiegelabbildungen schafft, auf welchen ein Kind (Mars) aus einem Gefäß mit heißem Wasser gezogen wird.

4 Dabei erwähnen Plutarch (Poplic. 2 I) und auch Zosimus (2, 3, 3) übereinstimmend, dass Poplicola dem Hades (Plutarch), beziehungsweise dem Dis und der Proserpina (Zosimus) geopfert habe. Diese Erwähnung der Unterweltsgötter in den Opfern von Poplicola nimmt Wagenvoort (1956) 206f. auf, wenn er wegen der gemeinsamen heiligen Tiere Specht und Wolf einen Kultzusammenhang zwischen Mars und der etruskischen Unterweltsgöttin Feronia (Proserpina) vermutet.

5 Um die ludi saeculares in Zusammenhang mit Mars stellen zu können, weist Wagenvoort (I956) 22 Iff. darauf hin, dass Mars mehrere Leben durchlaufen konnte, weswegen sich um ihn ein Wiedergeburtsmythos gebildet habe. Dies bekräftige ein Zitat von Aelianus, wonach der Kentaure Mares dreimal gelebt habe und dreimal gestorben sei (Ael. VH 9, I6). Dabei soll er jedes Mal I 23 Jahre gelebt haben, so lange, wie nach Cens. I7, 6 das fünfte saeculum der etruskischen Säkularberechnung gedauert haben soll. Dazu Wagenvoort (I956) 22 Iff.; ebenso Versnel (I982) $223 \mathrm{ff}$. 
verstehen. ${ }^{6}$ Wir müssen uns damit abfinden, dass wir über Feiern, die nachträglich in die Tradition der Säkularfeiern gestellt wurden, keine sicheren Aussagen machen können. Es bleibt weiterhin verborgen, ob vor den als historisch gesicherten Säkularspielen auf dem Tarentum das Heiligtum einer den Nachwuchs schützenden Gottheit ${ }^{7}$ oder irgendetwas anderes bestanden hat.

Der religiöse Grundgedanke der augusteischen ludi saeculares ist also nicht durch Rückgriff auf eine Ursprungsmatrix von Säkularfeier zu erfassen, sondern durch eine Interpretation der politischen und gesellschaftlichen Bedingungen, die Einfluss auf das religiöse Leben der Jahre um I7 v. Chr. hatte. Das bedeutet nicht, dass das Erfassen des religiösen Grundgedankens der Säkularfeier völlig unmöglich ist. Im Fall der Säkularspiele sind wir in der besonders günstigen Lage, über die Zeitgeschichte gut unterrichtet zu sein, durch zwei Historiker die zugrundeliegende Kultlegende gleich doppelt überliefert zu haben, daneben die rituelle Praxis durch die augusteischen und severischen commentaria genau zu kennen. Bei der Überlieferung der Kultlegende handelt sich um eine Schilderung des Ursprungs der Säkularfeier bei Valerius Maximus und bei Zosimus. ${ }^{8}$

Diese Überlieferungssituation ist typisch für diese Art römischer Erzählungen. Die Überlieferung von Kultlegenden ist ausschließlich im Bereich der Gelehrtenliteratur, insbesondere der Geschichtsschreibung und der gelehrten Dichtung, angesiedelt. Sie führte verbunden mit einer allgemein schlechten Quellenlage dazu, einen Zusammenhang zwischen ritueller Praxis und diesen Legenden zu leugnen oder zu ignorieren. ${ }^{9}$ Die Uneinheitlichkeit der Überlieferung römischer bezie-

6 Eine solche umfassende Interpretation liegt mit den Studien von Scholz (1970) zum altitalischen und altrömischen Marskult und Marsmythos vor. Scholz nimmt die seit dem I9. Jahrhundert geführte Diskussion über die Frage, ob Mars ein Kriegsgott oder ein umfassenderer omnivalenter Gott war, auf und unterstützt die Interpretation von Mars als einen in der Stadt Rom verehrten Gott, der mehr als nur Kriegsgott war. Dagegen und gegen die spekulative Methode von Scholz stellt sich Dumézil (1974) 2 I 5 ff. Für Dumézil bleibt Mars nach der Interpretation aller frühen topografischen, literarischen und epigrafischen Zeugnisse eindeutig ein Gott, der in Beziehung zum Krieg stand.

7 Diese Meinung vertritt La Rocca (1984) I 2 ff. Da La Roccas These stark von dem Bestreben geprägt ist, in den augusteischen Spielen keinen Bruch zu sehen, versucht er, die an der augusteischen Feier verehrten Göttinnen, Moiren, Ilithyien und Terra Mater, in Beziehung zu einem ursprünglichen Gentilkult der Valerier zu setzen. Ausgangspunkt seiner Überlegungen ist die Textkonstitution des lapis Satricanus. Er identifiziert den Gentilkult der Valerier als einen einer Fruchtbarkeitsgottheit geltenden Kult. Aronen (1989) I9-39 unternimmt einen Versuch, den Gentilkult der Valerier mit einem Faunuskult zu identifizieren. Dabei mag die Darstellung der gens Valeria als sabinische gens, die mit dem Mythos der ludi saeculares einen Ankunftsmythos ihrer gens in Rom und einen Ursprungsmythos für ihren Gentilkult besaß, zwar richtig sein, die Beziehungen des Gentilkultes zu Faunus ist jedoch noch weniger nachvollziehbar als alle anderen Identifikationen dieses Kultes.

8 Val. Max. 2, 4, 5; Zos. 2, I-3.

9 Einen Überblick über die Forschungsgeschichte zum römischen Mythos gibt Graf 
hungsweise italischer Mythen macht es unmöglich, auf den exakten Ursprung einer Erzählung zu schließen. Zu den überlieferungstechnischen Schwierigkeiten treten der Zentralismus Roms, der viele nichtrömische Ursprungsmythen überdeckte, und die Dominanz der griechischen Mythografie in Rom. Wegen dieser Überlieferungssituation wurde den Legenden oft eine politische Funktion zugewiesen, indem sie als nachträgliche Erklärungen oder gar Erfindungen interpretiert wurden, die einer bestimmten Ideologie verpflichtet waren. Dadurch wurden die Ursprungsmythen für die Erkenntnis einer Beziehung zwischen Kultlegende und Kult wertlos, weil sie einen Rückschluss auf eine Urfeier nicht mehr zuließen. Wenn es sich nun aber, wie im Fall der Säkularspiele, als unmöglich erweist, auf eine Ursprungsfeier zurückzugreifen oder diesen Kult für die voraugusteische Zeit zu rekonstruieren, so bleibt keine andere Möglichkeit, als den Mythos der Säkularspiele, so wie er uns überliefert ist, in Beziehung zu den Riten der augusteischen Säkularspiele zu setzen, so wie sie in den Quellen dargestellt sind. Das bedeutet, davon auszugehen, dass es sich bei diesem Mythos wahrscheinlich um eine Erzählung aus einem anderen Bereich handelte, die aus noch zu untersuchenden Gründen geeignet war, als Ursprungsmythos der Säkularspiele eingesetzt zu werden. Die augusteischen ludi saeculares bieten sich mit ihrer besonderen Überlieferungssituation geradezu an, die Rolle genauer zu untersuchen, die ein Mythos für die rituelle Praxis spielte. Wir sind nämlich über die »Tatsachen des Kultes, seine Zeiten, Lokale, Formen und Denkmäler « durch die inschriftlichen commentaria ausgezeichnet unterrichtet und besitzen außerdem sogar eine zweifache Überlieferung des Mythos. ${ }^{10}$

Der Mythos der ludi saeculares erschien nicht direkt an der Feier, sondern er spielte am Rande der Feier indirekt eine Rolle. Niemals wurde er in den Worten und Gesten der Feiernden, die uns in den commentaria überliefert sind, erwähnt, kein Dokument der Feier macht irgendeine Anspielung auf den Mythos. Seinen Ausdruck fand der Mythos an der Säkularfeier selbst wohl vorwiegend in den an die Opfer anschließenden ludi scaenici. ${ }^{11}$ Für uns ist der Mythos deshalb nur im Bereich der gelehrten Bücher und der Exegese der Feier wahrnehmbar, deren Quellen jedoch beträchtlich später aufgezeichnet wurden, als der Zeitpunkt der augusteischen Säkularfeier war. Um die Beziehung Mythos - Ritus betrachten zu können, will ich im Folgenden zuerst die zwei unterschiedlichen Darstellungen des

(1993a) 25-43. Die von Graf dargestellte Einschätzung römischer Mythen trifft auch für den Mythos der Säkularspiele zu, wenn er einerseits von Latte (I960) 246, Anm. 2 als »sentimentale Geschichte«, die »in maiorem gentis Valeriae gloriam erfunden« worden sei, dargestellt wird, andererseits von Wagenvoort (1956) I96ff. als eine Legende, durch die wir »details to the origin of the ludi Tarentini erhalten.

ı。 Wissowa (1904) I 29 bezeichnet die Kenntnis dieser Tatsachen als grundlegend dafür, um irgendetwas über die Bedeutung einer Kultlegende für die rituelle Praxis aussagen $\mathrm{zu}$ wollen.

i I Vgl. den Sachkommentar zu Z. I00-I02, S. I 34 f. 
Mythos selbst vorstellen, bevor ich auf die Zusammenhänge im Einzelnen eingehe. ${ }^{\text {I2 }}$

Ein reicher und angesebener Mann im Sabinervolk, namens Valesius, von dem das Geschlecht der Valerier abstammt, wohnte in seinem Landhaus in der Nähe des Ortes Eretum. Vor diesem Haus hatte er einen Hain mit sebr hohen Bäumen. Als infolge eines Blitzschlags diese vollständig ausbrannten, war Valesius sich nicht sicher, was dieses Zeichen mit dem Blitz zu bedeuten habe. Nun wurden seine Kinder, zwei Knaben und ein Mädchen, von einer Pest, die Stadt und Land heimgesucht hatte, befallen. Keiner der herbeigerufenen Ärzte konnte sie heilen, so suchte er Hilfe bei Wabrsagern. Diese interpretierten den Blitz als ein Zeichen göttlichen Zorns. Valesius versuchte deswegen die Götter durch ein Sübneopfer gnädig zu stimmen. Doch er und seine Frau waren dennoch weiterhin voller Angst und beide rechneten mit dem Tod der Kinder. Als Valesius vom Herd warmes Wasser für die Kinder holte, kniete er nieder und bat die Hausgötter (Hestia-Vesta) ${ }^{13}$, statt der Kinder ihn und seine Frau der Gefahr auszusetzen, die jetzt die Kinder bedrohte. Als er wieder in seinem vom Blitz getroffenen Hain arbeitete, glaubte er eine Stimme zu hören, die ihm die Heilung der Kinder versprach. Er solle die Kinder auf dem Tiber nach Tarent bringen und ihnen dort Tiberwasser zu trinken geben, das er auf einem Altar des Dis Pater und der Proserpina erwärmen solle. Diese Worte verstärkten seine Zweifel an der Rettung der Kinder und seine Verwirrung, weil man ihm eine lange und gefährliche Seereise befahl. Tarent liege ja an den Grenzen Italiens und außerdem dürfte wobl dort kein Tiberwasser vorhanden sein. Auch das Wort, er solle das Wasser auf dem Altar der Unterweltsgötter erwärmen, erfüllte ihn mit schlimmen Abnungen. Obwobl daraufhin auch die Wabrsager nicht mehr weiterwussten, befragte Valesius sie noch einmal und nun erkannte er, dass er der Gottheit gehorchen musste. Er setzte also seine Kinder am Tiberufer in ein Boot, das für die Flussfahrt geeignet war, und fuhr den Fluss mit der Strömung hinab in Richtung Ostia. Weil die Kinder wegen der Hitze zu fiebern anfingen, landete der Vater an einer Stelle, wo das Wasser rubig dabinzuziehen schien. Gegen Abend landete er in der Nähe des Marsfeldes, wo er ausstieg und den durstigen Kranken Wasser geben wollte. Da auf dem Boot kein Feuer vorhanden war, hatte ihn der Steuermann auf aufsteigenden Rauch hingewiesen. Er verbrachte die Nacht mit den Kleinen in der Hütte eines Hirten. Der Steuermann sagte ihm (Valesius glaubte eine Stimme zu hören), dass er in Tarent aussteigen solle, denn das sei der Name des Ortes und hatte damit die gleiche Bezeichnung wie jenes Tarent, das am Japygischen Vorgebirge liegt. Valesius dankte der Gottheit auf Knien für dieses glückliche Zusammen-

I 2 Ich übernehme das von Wuilleumier (1932) I 3 Iff. angewandte Darstellungsverfahren des Mythos: Normale Schrift stellt die bei Valerius Maximus und Zosimus übereinstimmenden Elemente der Geschichte dar, Fettdruck gibt die nur bei Valerius Maximus erwähnten Elemente wieder, kursiv gedruckter Text die nur bei Zosimus erwähnten Elemente. In den seltenen Fällen, in denen die beiden Autoren einander widersprechende Aussagen machen, sind beide Aussagen erwähnt, eine davon in runden Klammern. Eine Gegenüberstellung der beiden Originaltexte liefert Pighi (I94I) 4752.

I $3 \mathrm{Zu}$ dieser Differenz kommt es durch ein Übersetzungsproblem bei Zosimus. Bei Valerius Maximus, wie wahrscheinlich in allen anderen lateinischen Quellentexten, sind die Lares erwähnt. Da Zosimus kein griechisches Äquivalent im Plural kannte, aber dennoch die Verbindung mit dem häuslichen Herd betonen wollte, hat er in seiner Erzählung 'Eơía gewählt. Dazu Aronen (1989) 24 und 28. 
treffen und wies den Steuermann, anzulegen. Im Glauben, die Spur des von den Göttern genannten Heilmittels gefunden zu haben, schöpfte er mit einem Krug Wasser aus dem Fluss. Er erwärmte das Wasser auf einem von ihm errichteten Altar (Valesius trug das Wasser zu der Stelle, wo der Boden stärker rauchte, als dass man dies auf Reste eines Feuers zurückführen könnte. Im Vertrauen auf die Vorzeichen sammelte er allerlei brennbares Material und fachte durch ausdauerndes Blasen das Feuer wieder an). Als das Wasser warm war, gab er es den Kindern zu trinken, die daraufhin einschliefen und plötzlich von ihrer langen Krankheit gesund wurden. Die Kinder erzählten ihrem Vater, dass sie im Traum einen großen Mann von göttlicher Gestalt gesehen hätten, der sie mit einem Schwamm abgerieben hätte und ihnen befohlen hätte, dem Dis Pater und der Proserpina auf ihrem Altar, von wo sie das Wasser erhalten hatten, schwarze Opfertiere zu schlachten und an drei aufeinander folgenden Nächten auf dem Marsfeld, an dem Ort, der auch Tarentum beiße und wo sich auch eine Babn für Pferderennen befindet, Gesänge und Chöre aufzufübren (Göttermahle und nächtliche Spiele abzuhalten). Weil Valesius nirgends einen Altar gesehen hatte, wollte er einen Altar errichten und begab sich in die Stadt, um dort einen zu kaufen. Während jedoch die Steinmetze den Boden für das Fundament aushoben, fanden sie dort in einer Tiefe von 20 Fuß einen bereits fertigen Altar, auf dem geschrieben stand: »Dem Dis Pater und der Proserpina«. Als Valesius von einem Sklaven benachrichtigt worden war, ließ er sein Vorhaben, einen Altar zu kaufen, fallen. Jetzt wusste er genaner, was er zu tun hatte: Er schlachtete auf dem Tarentum schwarze Opfertiere, die von den Alten »furvus - dunkelfarbig « genannt wurden, und hielt an drei aufeinander folgenden Nächten Feiern (Spiele und Göttermahle) ab, weil genauso viele Kinder der Gefahr entronnen waren. Dieser Altar und der Ritus der Opferbandlung sind aus folgendem Anlass entstanden: Wäbrend des Krieges zwischen Römern und Albanern, als sich die beiden Völker schon bewaffnet gegenüberstanden, erschien ein Mann von riesenhaftem Aussehen, in ein schwarzes Fell gebüllt, und schrie laut, Dis Pater und Proserpina hätten befoblen, ibnen vor Eintritt in den Kampf ein unterirdisches Opfer darzubringen. Nach diesen Worten verschwand er. Die Römer aber gerieten über diese Worte in Unrube und bauten einen unterirdischen Altar, verrichteten auf ihm ein Opfer und bedeckten den Altar mit 20 Fuß hoch aufgeschütteter Erde, damit keiner, der nicht Römer war, davon Kenntnis hätte. Diesen Altar hatte Valesius gefunden, hier bielt er das Opfer und die nächtlichen Feiern ab und bekam deshalb den Namen Manius Valerius Tarentinus: Die unterirdischen Götter werden nämlich von den Römern Manen genannt, und "gesund sein " heißt bei ibnen "valere"; Tarentinus schließlich kommt von dem Opfer, welches er auf dem Tarentum dargebracht hat. In späteren Zeiten, im ersten Jahr nach der Königsherrschaft, befiel eine Pest die Stadt. Der erste Konsul, Poplicola, wollte seinen Mitbürgern helfen und legte an demselben Altar ein Gelübde ab, einen schwarzen Stier dem Dis Pater zu opfern und eine schwarze Färse der Proserpina und während dreier Nächte Göttermahle und Spiele zu feiern. Er ließ auf den Altar schreiben: "Ich, P. Valerius Poplicola, habe die weizentragende ${ }^{\mathrm{I}}$ Flur dem Dis Pater und der Proserpina geweibt und dem Dis Pater und der Proserpina zu Ehren für die Befreiung der Römer Spiele eingerichtet." Den Altar ließ er anschließend wieder mit Erde zuschütten, wie er vorher war.

I4 Bennett Pascal (I979) 533-536 weist auf ein Missverständnis in der Übersetzung des

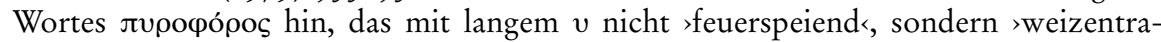
gend <edeutet: Valerius Maximus hat als Nichtgrieche das Wort mit den falschen Quantitäten gelesen und deshalb als $>$ feuerspeiend missverstanden. 
Als Erstes stellt sich die Frage, woher die beiden Autoren, Valerius Maximus und Zosimus, diese Erzählung übernommen haben. Die zahlreichen zusätzlichen Informationen bei Zosimus zeigen, dass Zosimus nicht, beziehungsweise nicht nur, von Valerius Maximus abhängen kann, die vielen Übereinstimmungen auch in Details lassen eher auf eine gemeinsame Quelle schließen. Als diese gemeinsame Quelle wurde meistens die Abhandlung über den Ursprung des Theaters von Varro gesehen, der wiederum von nicht mehr mit Sicherheit rekonstruierbaren Quellen abhängt, zu denen aber mit großer Wahrscheinlichkeit Valerius Antias gehörte. ${ }^{\text {Is }}$ Dieser Vertreter der jüngeren Annalisten aus dem ersten Jahrhundert v. Chr. ist bekannt für sein Bemühen, den Ruhm der gens Valeria in der Frühzeit Roms hervorzuheben, ein Zusammenhang, in welchen die Überlieferung des Mythos von Valesius gehören könnte. Das bedeutet, dass die Legende schon vor ihrer Verwendung als Kultlegende der ludi saeculares bestand und mit der gens Valeria verbunden war. Das äußerst vage Aufspüren der Herkunft dieses Mythos ist allerdings nicht das Entscheidende für das Verständnis, warum dieser Mythos in Verbindung zu den augusteischen Säkularspielen überliefert ist oder warum er zur Kultlegende der ludi saeculares gemacht werden konnte. Wichtig ist vorläufig, von seinem Inhalt und der Art seiner Verbreitung ausgehend seine Wirkung und Funktion $\mathrm{zu}$ bestimmen. ${ }^{16}$

Ohne dass wir also die Herkunft des Mythos genau bestimmen können, sollen doch wichtige Elemente des Mythos, in denen auf geografische, genealogische und kultische Realitäten der Riten der ludi saeculares eingegangen wird, verfolgt werden:

I. Die Opfer gehen ursprünglich von der gens Valeria aus, die sabinischen Ursprungs ist.

2. Die Opfer reagieren auf eine gesundheitliche Bedrohung der Nachkommenschaft dieser gens.

3. Der Ort der Opfer ist von göttlicher Seite befohlen und befindet sich am Tiberufer, dem späteren Terentum.

Is Blumenthal (I9I8) 220; Nilsson (I920) I70Iff.; Pighi (I94I) 4If. Diese Annahme beruht darauf, dass die Angaben bei Valerius Maximus über die Ursprünge verschiedener Spiele der Römer auf Varro, de scaenicis originibus zurückgehen. Alle drei Autoren räumen aber ein, dass Varro seine Informationen über die Säkularspiele durch Valerius Antias erhalten haben könnte, der möglicherweise Zugang zu einem Familienarchiv der Valerier hatte. Mit Sicherheit wird Antias bei Censorinus als Quelle für ludi saeculares erst für die Spiele von 249 v. Chr. angegeben, da der Text mit einer entsprechenden Quellenangabe für die früheren Spiele korrupt ist. Vgl. Cens. 17, Io.

I6 Burkert (1993) I 9f. weist darauf hin, dass eine Datierung von Mythen überhaupt nicht möglich und auch nicht sinnvoll sei, weil sie ständig an die jeweilige Realität angepasst wurden und somit die »Urform« eines Mythos nicht existiert. Wichtig sei dagegen, die Rezeption und Wirkung eines Mythos zu erfassen, um etwas über seine Funktion aussagen zu können. 
4. Dort soll sich ein unterirdischer Altar befinden, den eine Inschrift als Altar des

Dis Pater und der Proserpina ausweist.

5. Die Opfer für Dis Pater und Proserpina, die Valesius im Tiberknie begeht, bestehen aus drei dunklen Schafen und finden in drei aufeinander folgenden Nächten statt, Spiele schließen diese Opfer ab.

Die Teilnahme von zwei Mitgliedern der gens Valeria, (M.? Valerius) Potitus Messalla $^{17}$ und M. (Valerius) Messalla Messallinus ${ }^{18}$, an der augusteischen Säkularfeier wird in Zusammenhang mit den prosopografischen Untersuchungen noch einmal dargestellt. ${ }^{19}$ Im Mythos der ludi saeculares ist es ein Vertreter der gens Valeria, der einen Kult am Tiberufer für seine gens ausführt. Dies führte zu der Vermutung, dass es sich bei dem Kult der Säkularspiele ursprünglich um einen Gentilkult der Valerier handelte, der allein den Erhalt der Nachkommenschaft dieser gens betraf und durch Poplicola auf das römische Volk erweitert wurde. ${ }^{20}$ Die gens Valeria spielte nach der Überlieferung bei der Ausübung eines Kultes am Tiber immer eine wichtige Rolle: In der mythischen Zeit soll es ein Valerier gewesen sein, der den Kult begründet, in der Gründungszeit der Republik ist es angeblich wiederum ein Valerier, Valerius Poplicola, der als Konsul einen Kult zum Wohl der Nachkommenschaft der ganzen Stadt begeht, und in historischer Zeit sind es oft Konsuln aus der gens Valeria, unter deren Herrschaft Spiele ausgerichtet werden, die als Säkularspiele überliefert werden. ${ }^{21}$ Mit einer Interpretation dieses Mythos ausschließlich als Ursprungsmythos der ludi saeculares wird in einseitiger Weise die aitiologische Funktion des Mythos hervorgestrichen. Der Mythos aber sollte den Feiernden nicht erklären, warum die ludi saeculares gefeiert wurden. Er sollte vielmehr den religiösen Grundgedanken der Feier ausdrücken und anschaulich machen. ${ }^{22}$ Diesem Zweck diente der Inhalt des Mythos, in welchem ein Mitglied der gens Valeria den Teilnehmern und Teilnehmerinnen der augusteischen Säkularspiele beispielhaft die Sorge um seine bedrohte Nachkommenschaft vorführt. Damit wurde ein Problem der gesellschaftlichen Realität der augusteischen Zeit aufgenommen, nämlich die Bevölkerungspolitik. ${ }^{23}$ So gesehen spielte die gens Valeria nur eine exemplarische Rolle für die ludi saeculares: Ein Mythos, der in Beziehung zu ihrer gens stand, fand auch für die Aussage der Säkularfeier Verwendung - möglicherweise mit verschiedenen Anpassungen und Erweiterungen

I7 Z. I50, I54.

I 8 Z. I 52.

i9 Vgl. Kap. A.8, S. 227 zur Aufnahme von M. Valerius Messalla Messallinus in das Kollegium.

20 Aronen (I989) 23f. versteht den Mythos außer als Begründungmythos für den Kult auf dem Terentum auch als Ankunftsmythos der sabinischen gens Valeria in Rom.

2 I Vgl. die Tabelle S. I7 I mit den Konsuln der einzelnen Spiele.

22 Vgl. Scheid (i993b) i i $3 \mathrm{f}$.

23 Vgl. S. 268 f. zum Bevölkerungsstand im Jahre i7 v. Chr. 
der Erzählung. Die Valerier haben nicht den Kult der Säkularspiele begründet, sondern sie haben ein Verhalten gezeigt, das für alle Römer und Römerinnen als nachahmenswert gelten sollte.

Im Mythos hat Valesius das Opfer nach der Gesundung seiner drei Kinder ausgeführt. ${ }^{24}$ Die augusteischen Säkularspiele haben den Gedanken des Erhalts der Nachkommen in besonderer Weise betont, so sehr, dass gewisse Interpreten gerade diese Ausrichtung der Feier ausschließlich als aufgesetzte augusteische Propaganda verstanden haben oder als unverständlichen Rückgriff auf alte Erscheinungen der römischen Religion. ${ }^{25}$ Es stellt sich in der Tat die Frage, was der Erhalt der Nachkommenschaft mit dem religiösen Grundgedanken der Säkularfeier zu tun hat, also mit dem Ablaufen eines saeculum und dem Beginn des nächsten. Auf den ersten Blick weist kein einziges Motiv des Mythos auf ein zyklisches Vergehen von Zeit hin. Dieses Problem wurde in der bisherigen Behandlung der ludi saeculares wohl auch aus diesem Grund unbeantwortet gelassen. ${ }^{26} \mathrm{Um}$ den religiösen Grundgedanken der Säkularfeiern zu erfassen, hat sich als Erster Diehl mit der Etymologie des Wortes saeculum beschäftigt. saeculum wird seit Diehl von saitlo = Generation abgeleitet. ${ }^{27}$ Es handelt sich bei saeculum also nicht um ein chronologisches Maß von einer genau festgelegten Anzahl Jahren, sondern um einen Lebenszyklus, dessen Maß durch eine in ihm lebende Generation bestimmt wird. Darauf verweist auch die etruskische Vorstellung von einem saeculum, das dann abgelaufen ist, wenn keiner mehr lebt, der bei Beginn dieses saeculum schon am Leben war. Diese Vorstellung ist in die Säkularfeiern eingeflossen, insofern dass kein Mensch mehr als einmal in seinem Leben Säkularspiele erleben kann. ${ }^{28}$ Unter dieser Voraussetzung war das Feiern von Säkularfeiern als Anlass gedacht, durch welchen eine Verbindung von einer Generation zur nächsten hergestellt werden konnte. Es handelte sich um ein Fest, das den Menschen bewusst machte, dass ihre Sache - bei den Etruskern: ihre Stadt - weiterhin Bestand haben würde, auch wenn die nun lebenden Menschen gestorben seien. Das Vorhandensein einer neuen Generation stellte die Kontinuität eines Unternehmens sicher - über die Generationen hinaus. Dieser Zusammenhang sollte durch den Mythos der Säkularspiele ausgeführt werden. Man kann wohl davon ausgehen, dass dieser Gedanke der

24 Der von Valerius Poplicola eingerichtete Kult reagierte ebenfalls auf eine Gefährdung der Nachkommenschaft der Stadt Rom. Plut. Poplic. 2I, I.

25 Zanker (1997) 3 172; für Latte (1960) 299 sind die Gottheiten der nächtlichen Opfer der augusteischen Säkularfeier »unbestimmt und gestaltlos, wie es sich für nächtliche Gewalten geziemt $\ll$.

26 Bernstein (1998) I 38.

27 Diehl (1934/35) 255 f. Ebenso Walde-Hofmann (I939) 460f.; die bei Freyburger (I 993) 95 vorgetragene semantische Untersuchung des Wortes kommt zu demselben Schluss und versteht saeculum als Generation, Rasse und Lebensdauer.

28 Dieses Motiv enthielt die Einladung der Herolde zur Säkularfeier: Zos. 2, 5, ı; Suet. Claud. 21, 5; Cens. I7, I3. Das augusteische commentarium enthält diese Vorstellung ebenfalls: Z. 54-56. 
Fortsetzung und Sicherung der Kontinuität ein Grundgedanke von Säkularfeiern war, den die augusteische Säkularfeier in besonderer Weise aufnahm, weil er der augusteischen Ideologie entsprach. ${ }^{29}$ Denn die in den drei Nächten der augusteischen Feier mit Opfern bedachten Göttinnen - die Moiren, Ilithyien und Terra Mater - haben alle eine den Nachwuchs schützende Funktion..$^{30}$ Das Auftreten der I IO Matronen in jeder Nacht und an jedem Tag der Feier mit sellisternia und Supplikationsgebet am zweiten Tag ist als Hinweis zu verstehen, dass die augusteische Politik für diese Aufgabe besonders die Frauen verantwortlich machte und sie deshalb in herausragender Weise an den Säkularspielen beteiligte. Auch der Chor der je 27 Knaben und Mädchen, der das carmen saeculare aufführte, kann als eine Form der Darstellung dieser Aussage der ludi saeculares verstanden werden. ${ }^{31}$ Die augusteischen ludi saeculares haben auf vielen Ebenen ausgeführt, dass das sichere Nachwachsen neuer Generationen das nächste saeculum garantieren wird; neben vielen rituellen Elementen war es gerade die Aufführung des Mythos, die diese Aussage in den ludi scaenici szenisch darstellte.

Die rituelle Praxis der augusteischen ludi saeculares zeigt als wichtiges Element die Wahl des Ortes, der außerhalb des pomerium lag und offenbar nur für diesen Anlass aufgesucht wurde. Für die Wahl dieses Ortes hat der Mythos der Säkularspiele eine aitiologische Funktion, denn er erklärt mit seiner Geschichte, wieso an einem so unzugänglichen und abgelegenen Ort wie dem Tarentum ein wichtiger öffentlicher Kult vollzogen wird. Es fällt auf, dass das augusteische commentarium als Austragungsort niemals vom Tarentum spricht, sondern immer von einem Ort ad Tiberim..$^{22}$ Diese Ausdrucksweise verweist auf die wichtige Funktion des Tibers, der auch im Mythos eine Rolle spielt und diese Gegend prägt. Es ist nicht mehr rekonstruierbar, in welcher Weise die unmittelbare Nähe des Flusses für die Spiele bedeutsam war, es fällt aber auf, dass einige domitianische Münzen den Flussgott Tiber abbilden. ${ }^{33}$ Die augusteische Inschrift hat vielleicht mit ihrer Ausdrucksweise ad Tiberim eine Verbindung zum Mythos herstellen wollen, indem sie die andere Bezeichnung des Ortes Tarentum vermied, die nur indirekt auf den Mythos anspielt. Es ist sogar möglich, dass die Bezeichnung Terentum oder Ta-

29 Vgl. S. $280 f$.

30 Z. $90 \mathrm{ff}$.; I I $5 \mathrm{ff}$; I $34 \mathrm{ff}$. Das severische commentarium belegt, dass auch an der severischen Säkularfeier diesen drei Göttinnen die nächtlichen Opfer ausgerichtet wurden: Act. Sev. I $38 \mathrm{ff}$. (Moiren); 172-176 (Ilithyien); 222-228 (Terra Mater). Außerdem sind auch sind im Orakel zu den Spielen diese Göttinnen erwähnt; siehe Pighi (I94I) 56 f., Verse 4-I I. Zu der kourotrophen Funktion dieser Göttinnen vgl. den Sachkommentar zu Z. $90 \mathrm{f}$, I I 5-I 8 , I 34 f.

3 I Vgl. Scheid (I993b) i I 3 .

32 Z. I08, II 5 , I34, I 57.

33 Zwei domitianische Münzen, die ein Opfer darstellen, zeigen den Gott Tiber: siehe Anhang, Abb. I I (Ilithyia-Opfer) und Abb. I 2 (Opfer für Terra Mater). La Rocca (1984) 24 weist darauf hin, dass Kulte geburtsfördernder Göttinnen oft eng mit Wasserläufen verbunden sind. 
rentum zu stark an das Heiligtum von Dis Pater und Proserpina gebunden war, deren Verehrung an der augusteischen Säkularfeier nicht vorgesehen war. ${ }^{34}$

Der bei Valerius Maximus überlieferte Mythos zeigt uns, dass zu dem Kult am Tiberufer neben nächtlichen Opfern ludi und lectisternia gehörten: hostias negras, quae antiquitus furvae dicebantur, Tarenti immolavit ludosque et lectisternia continuis tribus noctibus ... fecit. ${ }^{35}$ Auch wenn diese Darstellung bereits von der späteren Erscheinungsform des Festes geprägt sein wird, ist es doch wahrscheinlich, dass die Durchführung von Spielen nach den Opfern schon sehr früh zu diesem Kult gehörte, denn diese gaben dem Kult sogar den Namen als ludi saeculares. Auch der Kultort, der eng mit einer Wettkampfstätte verbunden war, verweist darauf. ${ }^{36}$ Das Opfer mit seinen besonderen Bedingungen und die anschließenden Spiele gehören zu einem rituellen Ensemble, das im Mythos der Säkularspiele erwähnt ist. ${ }^{37}$ Das bedeutet nicht, dass der Mythos dieses rituelle Ensemble erklärt, aber er schafft mit seiner Erzählung einen Rahmen, in welchen die rituellen Besonderheiten der ludi saeculares passen und in einer bestimmten Stimmung erscheinen. Der Mythos unterhält das Publikum und er regt die Vorstellungskraft der Teilnehmer der Säkularfeier an.

Über die Gestaltung dieser ludi sind wir im Einzelnen nicht unterrichtet. Es hat sich wahrscheinlich um szenische Darstellungen gehandelt, sogenannte frei improvisierte ludi scaenici, die zusammen mit vorher verfassten hymnischen Gesängen vorgetragen wurden. Darüber macht nur Zosimus spärliche Angaben. ${ }^{{ }^{8}}$ Als zusammengehörende kultische Einheit boten die ludi und die vorgetragenen Festgesänge die Möglichkeit, den Mythos, der der Säkularfeier zugrunde lag, zu erwähnen und darzustellen. Es ist wahrscheinlich, dass die Geschichte der Landung des Valesius mit seinen kranken Kindern am Tiberufer dramatisch gestaltet war und am Ort des Geschehens zur Aufführung kam. Da über die Entwicklung der ludi scaenici kaum etwas bekannt ist, können Vermutungen über die Art dieser Aufführungen nur spekulativ sein. ${ }^{39}$

34 Vgl. S. 205 f.

35 Val. Max. 2, 5.

36 Zur Wettkampfstätte, die sich an das Tarentum anschloss, dem trigarium, siehe S. 2 Iof.

37 Auch Zosimus (2, 2, 3) erwähnt in seiner Darstellung des Mythos die dreifache Durchführung von Opfern mit dunklen Opfertieren in drei Nächten und anschließende Feste und Gesänge.

38 Zosimus $(2,5,3)$ gibt als einzige Quelle einen Hinweis über den Inhalt dieser ludi:

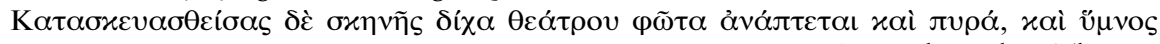

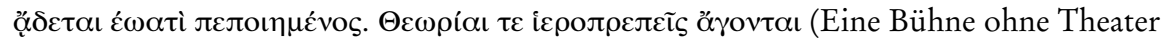
ist errichtet worden, Fackeln sind angezündet worden und ein eben erst verfasster Festgesang ist gesungen worden, außerdem sind Schauspiele aufgeführt worden, die dem Heiligtum angemessen waren). Vgl. den Sachkommentar zu Z. Io0-ıo2, S. I 34 ff.; Bernstein (1998) I 30.

39 Vgl. Bernstein (1998) i i $9 \mathrm{ff}$. 
Ein bisher unberücksichtigtes Motiv des Mythos ist das Vergraben des Altars und sein unerwartetes Auftauchen. Sowohl Valerius Maximus wie auch Zosimus berichten übereinstimmend davon: Valesius' Leute, die eine Grube für das Fundament des zu errichtenden Altars ausheben, stossen in der beträchtlichen Tiefe von 20 Fuß auf einen bereits fertigen Altar mit einer Inschrift, die diesen Altar als Besitz des Dis Pater und der Proserpina ausweist. ${ }^{\circ}$ Dieses unverhoffte Auftauchen des Altars und damit das Aufzeigen der Fälligkeit eines Opfers steht in Beziehung zu dem von den Etruskern überlieferten Grundmotiv der Säkularidee: Ein saeculum ist dann vorbei, wenn der letzte Mensch gestorben ist, der bei der Gründung einer Stadt schon am Leben war. ${ }^{4 \mathrm{I}}$ Daraus geht hervor, dass sich der Zeitpunkt, wann ein saeculum abgelaufen ist und Säkularopfer und -spiele fällig sind, der menschlichen Kenntnis entzieht. Der fällige Zeitpunkt wird den Menschen von göttlicher Seite durch portenta mitgeteilt. Ebenso entzog es sich nach dem Mythos der Kenntnis des Valesius, dass an dem Ort und zu dem Zeitpunkt, als er scheinbar zufällig im Tarentum landete, die Opfer an dem scheinbar zufällig ausgegrabenen Altar fällig waren. Der Zeitpunkt und der richtige Ort des Opfers wurden ihm offenbart. In ähnlicher Weise gehörte es zum Wesen der Säkularspiele, dass ihr Zeitpunkt angeblich nicht von Menschen festgesetzt werden konnte, sondern dass sie aufgrund göttlicher Zeichen fällig waren. Ob es auch für den Ort eine ähnliche Parallele gab, ist nicht mehr feststellbar; denkbar ist, dass das Gebiet am Tiber zwischen den Säkularfeiern durch Überschwemmungen und überwuchernde Vegetation derart verändert wurde, dass es bei der Vorbereitung einer neuen Feier nicht mehr als Kultgelände erkennbar war.

Ebenso bedeutsam ist die Angabe im Mythos, dass der ausgegrabene Altar eine Inschrift trug. Die inschriftliche Erinnerung an die begangene Feier und an früher begangene Feiern scheint zumindest seit der augusteischen Säkularfeier ein wichtiges Element gewesen zu sein..$^{42}$ Hinter dieser Notwendigkeit einer inschriftlichen Fixierung der ausgeführten Riten der Feiern steckt die Annahme, dass niemand mehr am Leben sei, der die letzte Säkularfeier erlebt hat. Aus diesem Grund müssen die nötigen Riten inschriftlich und dauerhaft und sicher der Nachwelt überliefert werden. Auch damit liegt ein Bezug zur etruskischen Säkularidee vor, die davon ausgeht, dass niemand mehr lebt, der über das Ablaufen eines saeculum Rechenschaft ablegen kann. Die Bedeutsamkeit dieses Motives bestätigen die

40 Auch bei Fest. p. 440 Lindsay wird dieser vergrabene Altar erwähnt; erstaunlicherweise ebenfalls mit der Angabe, dass er 20 Fuß unter der Erde vergraben war, aber ohne diesen Altar in Zusammenhang mit den Spielen des Valerius Poplicola zu bringen.

4I Cens. I7, 5 .

42 Auch Valerius Poplicola hat im Anschluss an die von ihm veranstalteten Spiele eine Inschrift auf den Altar setzen lassen, die der Nachwelt die von ihm begangenen Riten mitteilte. Die Inschrift auf dem Altar erwähnt nur Zosimus, Valerius jedoch schildert, dass der Altar nach den Riten wieder vergraben wurde. 
Münzprägungen anlässlich von Säkularspielen: Das Motiv der Inschrift ist nämlich in allen drei erhaltenen Münzprägungen zu Säkularfeiern aufgenommen. ${ }^{43}$ Andererseits ist auch gut vorstellbar, dass gerade dieses Motiv einer neuen medialen Ausrichtung der augusteischen Zeit entsprach, die vermehrt Monumentalinschriften aufstellen ließ. Die augusteische Inschrift weist explizit darauf hin, dass sowohl eine marmorne wie auch eine bronzene Inschrift AD CONSERVANDAM MEMORIAM TANTAE R/eligionis auf Staatskosten errichtet werden sollten. ${ }^{44} \mathrm{Ge}-$ rade an diesem ungewöhnlichen Aufwand der zweifachen Anfertigung einer Inschrift wird deutlich, wie ernsthaft die Organisatoren der augusteischen Feier dieses Motiv berücksichtigt haben. Es ist auch denkbar, dass dieses Motiv des Mythos von anderer Stelle in die Erzählung eingefügt wurde, um so ein aktuelles Medium der schriftlichen Fixierung als traditionell ausgeben zu können. Das Motiv einer unterirdischen Aufbewahrung der Inschrift ist allerdings für die augusteischen Spiele nicht mehr auszumachen. Dagegen spricht die zu geringe Tiefe des Fundortes der Fragmente. ${ }^{45}$ Nur die Ausradierung des Namens von C. Asinius Gallus (Z. I68) unter Tiberius zeigt, dass die Inschrift dreißig Jahre danach noch zugänglich war. Auch die severische Inschrift war nicht vergraben, weil sie sieben Jahre nach der severischen Säkularfeier, im Jahr 2 I I n. Chr., für die Rasur des Namens von Geta zugänglich war.

Nachdem die vielfältigen Beziehungen, die zwischen Mythos und ritueller Gestaltung der augusteischen Säkularfeier bestanden haben, dargestellt worden sind, ist es an der Zeit, die Funktion des Mythos allgemeiner zu bestimmen. Der Mythos erschien - wie wir gesehen haben - nicht in direkt sichtbarer Weise, das heißt, die Quellen erwähnen ihn nie in Zusammenhang mit den Riten der Feier. Demnach war auch ohne Kenntnis dieses Mythos das Begehen der Säkularfeier möglich. Es ist sogar davon auszugehen, dass der Mythos den meisten der Feiernden nicht oder nicht vollständig und genau bekannt war, denn seine Überlieferungstradition stellt ihn eindeutig in den Rahmen der römischen Gelehrsamkeit. Der Mythos diente also nicht dazu, den Feiernden die vollzogenen Riten in irgendeiner Weise einsichtig zu machen oder zu erklären. ${ }^{4}$ Dies war auch nicht nötig, denn Riten müssen nicht verstanden werden, sie erhalten ihre Existenzberechtigung nicht dadurch,

43 Für die augusteische Feier siehe Anhang, Abb. 3, für die domitianische Feier Abb. 6 und 7, für die severische Feier Abb. I9.

44 Z. 58-63.

45 Die Fragmente der augusteischen Inschrift waren in einer mittelalterlichen Befestigungsmauer des Tibers verarbeitet worden. Es ist denkbar, dass die Fragmente ursprünglich unterirdisch lagerten, aber im Laufe der Zeit durch eine vollständige Umgestaltung des Geländes wieder ans Tageslicht gekommen waren, sodass sie zur Zeit ihrer Verwendung für die mittelalterliche Mauer offen lagen. Es ist aber unwahrscheinlich, dass sie - wie Zos. 2, 3, 2 erwähnt - in einer Tiefe von 20 Fuß (immerhin mehr als $6 \mathrm{~m}$ ) lagen, weil diese Tiefe kaum ein Auftauchen der Inschrift durch Umgestaltung des Geländes erlaubt. Vgl. S. 208.

46 Scheid (I993b) II4. 
dass sie erklärbar sind, sondern dadurch, dass sie gemeinschaftlich und wiederholt vollzogen werden. Dem Mythos der augusteischen Säkularfeier kam also für die Feiernden nicht in erster Linie eine aitiologische Funktion zu.

Die Funktion des Mythos war nicht eindeutig festgelegt. Er existierte im Hintergrund der Feier und trug dazu bei, die Feier in einer bestimmten Weise auszustatten. Wahrscheinlich war es die Kraft des Mythos, die eine Feier in einen Interpretationsrahmen stellen konnte, der von den Organisatoren der Feier beabsichtigt war. Die Kraft des Mythos erschien den Feiernden nicht direkt in den praktizierten Riten, sondern in einer bestimmten Stimmung, die von der Art, wie die Riten zelebriert wurden, ausging. Die Stimmung der nächtlichen Opfer, das Auftreten der i io Matronen zu den sellisternia nach jedem Opfer, der von der Stadt abgelegene, sonst nie besuchte Ort des Tarentum, die an die Opfer anschließenden ludi, der Vortrag des carmen saeculare durch je 27 Knaben und Mädchen waren Elemente, in denen es möglich war, Motive des Mythos zu erkennen. Diese Elemente waren es, die die Feier für die Teilnehmer nicht als irgendwelche Opfer erscheinen ließen, sondern als das im Leben einmalige Ereignis einer Säkularfeier. Es ist davon auszugehen, dass uns viele weitere Elemente, die vielleicht in der Kleidung oder Gestik der Beteiligten zum Ausdruck kamen, unbekannt sind. Deswegen sind unsere Kenntnisse über den römischen Mythos auch so beschränkt, weil sein Erscheinungsbild außerordentlich frei war und in einem Bereich stattfand, der nicht überliefert ist. Das Erscheinungsbild des Mythos war äußerst wandelbar und an die jeweilige Situation anpassbar.

Der Mythos diente also dazu, die sehr starren Riten des römischen Opfers und Gebets zu differenzieren. Bei einem Vergleich der Säkulargebete mit den Gebeten in Catos De agricultura zeigt sich die formale Gleichheit römischer Gebete, obwohl es sich bei Cato um Gebete in einem völlig anderen Zusammenhang handelt. ${ }^{47}$ Durch die an die Opfer anschließenden ludi, die den Mythos darstellten und rituell mit den Opfern zusammengehörten, wurden die vorangegangenen Opfer und Gebete als Säkularopfer und Säkulargebete wahrgenommen. Der Mythos stellte Riten, die an jeder religiösen Feier ähnlich zu sein schienen, in den Zusammenhang eines besonderen Anlasses. Gerade in dieser Differenzierung der Opfer und Gebete lag für die Feiernden wahrscheinlich ein Moment der Erbauung und Unterhaltung, welches die sonst ewig gleichen religiösen Riten belebte und erfreulicher machte. Für die Organisatoren der Säkularfeier lag in dieser Funktion des Mythos ein Moment der ästhetischen Gestaltung und der Möglichkeit, auf den Zeitgeschmack einzugehen. Somit wird dem Mythos die wichtige Funktion zugekommen sein, unter den Feiernden einen consensus herzustellen. Dieser consensus betraf keine inhaltlichen oder religiösen Fragen, sondern den gesamten ästhetischen Gestaltungsrahmen eines religiösen Festes. Der Mythos begleitete die Riten, schmückte sie aus und ermöglichte durch seine Erscheinungsweise Vorfreude und 
Freude an den Riten. Andererseits begleiteten die Riten den Mythos, indem sie die Darbietungen des Mythos über ein reines Unterhaltungsprogramm erhoben und ihn durch seine Einbettung in die rituelle Praxis $\mathrm{zu}$ einem wichtigen gemeinschaftlichen religiösen Akt werden ließen.

Auf diese Weise erhielt der Mythos mit den oben beschriebenen Ausrichtungen eindeutig eine politische und soziale Funktion, die Möglichkeiten des Missbrauchs bot. Aber auch damit steht der Mythos in der Tradition der römischen Religion, deren Angelpunkt der Interpretation nicht das Leben der Götter im Olymp war, sondern das Leben der Römer in Rom. Es ging nicht darum, Fragen des menschlichen Zusammenlebens in einem allgemeinen Sinn zu reflektieren, sondern darum, die jeweilige historische Situation Roms zu reflektieren. Zur Zeit der augusteischen Säkularspiele war der Mangel an Nachwuchs in den senatorischen Familien offenbar ein zentrales Problem, sodass dieser Aspekt an der augusteischen Säkuarfeier verstärkt seinen Ausdruck fand..$^{8}$ Man kann aber nicht sagen, dass die augusteische Feier diesen Aspekt vollkommen neu erfunden hätte. Dies zeigt die Wandelbarkeit des Mythos, der in unterschiedlichen Aspekten aufgenommen werden konnte und der sich im Laufe seiner Erzählung durch Anpassungen immer wieder veränderte. Möglicherweise ist ein Detail des Mythos, das nur bei Valerius Maximus überliefert ist, eine solche augusteische Aktualisierung: Valerius Maximus berichtet, dass es sich bei den drei Kindern des Valesius um zwei Knaben und ein Mädchen handelte. Zur Zeit der Säkularspiele hatte das Paar Iulia/Agrippa gerade drei Kinder: den 20 v. Chr. geborenen C. Caesar, die I9 v. Chr. geborene Iulia und den kurz vor den Säkularspielen geborenen L. Caesar. Wir können allerdings nicht mehr feststellen, inwiefern die Aufführungen des Mythos solche Parallelen augenfällig machten.

Und doch oder vielleicht sogar wegen seiner Wandlungsfähigkeit war eine weitere Funktion des Mythos, die Kontinuität einer Feier sicherzustellen. Der Mythos bot nämlich einerseits sehr unterschiedliche Aspekte an, die eine Feier aufgreifen konnte. Andererseits aber wurde mit dem Hintergrund des immer gleichen Mythos eine Kontinuität sichergestellt, die verhinderte, dass trotz gewisser Änderungen eine Feier nicht mehr als das ursprünglich gefeierte Fest erkannt wurde. Mit dieser vielfältigen Möglichkeit der Ausrichtung konnte eine religiöse Feier auf die jeweilige historische Situation reagieren, aber dennoch spezifisch als Säkularfeier wahrgenommen werden. Es ist nur zu vermuten, welches die Elemente waren, die unerlässlich waren, damit ein Fest als Säkularfeier eingeordnet wurde. Als eindeutigstes Element ist der Ort der Feier, das Tarentum, zu werten, denn der Ort wurde nur für diesen Anlass genutzt. Ebenso wichtig wird der Zeitpunkt der nächtlichen Opfer gewesen sein und vielleicht noch als drittes Element die Errichtung einer Inschrift. Solche ständig gleichbleibenden Motive, deren Zusammenhang mit dem Mythos als sicher gelten darf, prägten die Vorstellung darüber, was eine Säkular-

48 Jacques/Scheid (I998) 325. 
feier ist und stellten eine Kontinuität her. ${ }^{49}$ Sie ließen es zu, dass zum Beispiel die an den verschiedenen Säkularfeiern geehrten Götter nicht immer dieselben sein mussten. ${ }^{\circ}$ Da über die älteren Säkularfeiern keine Einzelheiten der rituellen Gestaltung bekannt sind, müssen Aussagen über den Wandel, den die Säkularfeier unter Augustus erfahren hat, eher vage bleiben. ${ }^{\text {sI }}$

Damit kann der Mythos zu den Säkularspielen neu eingeordnet werden: Es handelt sich auf keinen Fall um eine »sentimentale Geschichte«, mit der die augusteischen Rekonstrukteure der Säkularfeier aufwarteten, um der Feier einen zugänglicheren Charakter zu geben. ${ }^{22}$ Auch eine Interpretation des Mythos als Legende eines Gentilkultes der Valerier, die diese zum Ruhm ihrer eigenen Familiengeschichte verbreitet haben, trifft nicht den Kern dieses Mythos. Es handelt sich um eine Erzählung, die über den Kreis der gens Valeria schon früh hinausging und als Ursprungsmythos eines wichtigen öffentlichen Festes verwendet wurde. $\mathrm{Zu}$ welchem Zeitpunkt diese Verbindung hergestellt wurde, ist nicht mehr erkennbar. Der Mythos enthält einige aitiologische Momente, wichtiger aber ist, dass er an der augusteischen Säkularfeier der römischen Bevölkerung klarmachte, dass ihre Zukunft und Stärke in einer gesunden Nachkommenschaft liege. Damit erhält der

49 So wird ein Römer, der keinerlei religionshistorische Bildung hatte, doch gewusst haben, dass eine Säkularfeier auf dem Tarentum stattfindet. Darauf weist das Epigramm Martials ( 10,63 , 3 f.) hin: Die Grabinschrift will ausdrücken, dass die Verstorbene ein ungewöhnlich langes Leben hinter sich hat, das es sogar zuließ, zweimal in einem Leben auf das Tarentum zu kommen, was nur für eine Säkularfeier geschah (aus Anlass der claudischen - 47 n. Chr. - und domitianischen - 88 n. Chr. - Säkularfeier). Umgekehrt muss man daraus den Schluss ziehen, dass der Besuch des Tarentum nur in Verbindung mit einer Säkularfeier assoziiert wurde, die manch anderer überhaupt nicht erlebt hat.

50 Die Quellen der Säkularfeiern scheinen sich nicht in erster Linie mit den an der Feier geehrten Göttern beschäftigt zu haben. So äußert sich nur Varro bei Censorinus ( I 7, 8) darüber, dass die Opfer und Spiele dem Dis Pater und der Proserpina gelten. Alle anderen bei Censorinus angeführten Quellen machen keine Angaben über Götter. Ebenso werden die betroffenen Götter auch bei Zosimus für die republikanischen Säkularspiele nur mit Dis Pater und Proserpina angegeben, während er für die Angaben über die augusteischen Spiele wahrscheinlich auf die Inschrift zurückgreifen konnte und die dort verehrten Götter nennt, ohne eine Bemerkung über einen Wandel zu machen. Offenbar war das, was eine Säkularfeier ausmachte, nicht vorrangig ein Opfer an die Unterweltsgötter, sondern die Ausübung von nächtlichen Riten auf dem Tarentum. In keiner Quelle ist auch nur die leiseste Verwunderung darüber angedeutet, dass seit den augusteischen Säkularspielen andere Götter verehrt worden sind. Dies deutet darauf hin, dass die geehrten Götter nicht den Charakter der Säkularspiele prägten.

ऽ I Von einer völligen Umgestaltung der Feier, was vorwiegend in älteren Interpretationen der augusteischen Feier behauptet wurde, kann wohl nicht gesprochen werden. Die augusteischen Neuerungen waren einschneidend, solange uns aber der genaue Vergleich mit früheren Feiern nicht möglich ist, lässt sich das Ausmaß der Umgestaltung nicht bestimmen.

52 Vgl. S. I85f., Anm. 9. 
Mythos der ludi saeculares eine zweckgerichtete Note, die über Belehrung und Unterhaltung hinausgeht und einen »die Zukunft erzwingenden« Charakter hat. ${ }^{53}$ Der Mythos der Säkularfeier stellt einen Anspruch an die Feiernden, er verkündet ein Programm, das als Prägung der Realität gelten kann, nämlich die Erhaltung der Nachkommen. Für die augusteische Zeit erhielt diese Seite der Säkularfeier nach der Dezimierung der Bevölkerung während der Bürgerkriege eine dringende Aktualität. Der Mythos machte klar, dass die verantwortungsvolle Sorge um die Nachkommen in direktem Zusammenhang mit dem Wohl und Fortbestehen des Staates steht, um welches es an den Säkularspielen, die ludi publici waren, ging. Auf diese Weise lenkte der Mythos der Säkularspiele das religiöse Denken der Teilnehmenden - und zwar der Männer und in besonderer Weise auch das der Frauen - in eine bestimmte Richtung. Dieses Denken wirkte sich auf das persönliche Leben jedes Einzelnen aus und lieferte eine Begründung für die Mühen und Entbehrungen, die man mit der Aufzucht eigener Kinder auf sich nahm. Der Mythos erfasste damit die konkrete Wirklichkeit jedes Einzelnen, bot aber die Möglichkeit, die Wirklichkeit in einen größeren Zusammenhang zu stellen und sich als mitverantwortlich für ein gemeinsames Ziel zu verstehen.

53 Burkert (1993) i9. Die Gestalt des Valesius erfüllt eine exemplarische Funktion, ihn umgibt, wie Burkert sagt, »die Aura des Vorbildlichen«, dessen Verhalten für das augusteische Publikum Maßstäbe setzte. 